\title{
The AB Doradus system revisited: The dynamical mass of $\mathrm{AB}$ Dor $\mathrm{A} / \mathrm{C}$
}

\author{
R. Azulay ${ }^{1,2}$, J. C. Guirado ${ }^{1,3}$, J. M. Marcaide ${ }^{1}$, I. Martí-Vidal ${ }^{4}$, E. Ros ${ }^{2,1,3}$, E. Tognelli5 ${ }^{5,6}$, D. L. Jauncey ${ }^{7,8}$, \\ J.-F. Lestrade ${ }^{9}$, and J. E. Reynolds ${ }^{7}$ \\ 1 Departament d'Astronomia i Astrofísica, Universitat de València, C. Dr. Moliner 50, 46100 Burjassot, València, Spain \\ e-mail: Rebecca.Azulay@uv.es \\ 2 Max-Planck-Institut für Radioastronomie, Auf dem Hügel 69, 53121 Bonn, Germany \\ 3 Observatori Astronòmic, Universitat de València, Parc Científic, C. Catedrático José Beltrán 2, 46980 Paterna, València, Spain \\ ${ }^{4}$ Onsala Space Observatory, Space Earth and Environment, Chalmers University of Technology, 43992 Onsala, Sweden \\ 5 Department of Physics "E. Fermi", University of Pisa, Largo Bruno Pontecorvo 3, 56127 Pisa, Italy \\ 6 INFN, Section of Pisa, Largo Bruno Pontecorvo 3, 56127 Pisa, Italy \\ 7 CSIRO Astronomy and Space Science, Australia Telescope National Facility, Epping, NSW 1710, Australia \\ 8 Research School of Astronomy and Astrophysics, Australian National University, Canberra, ACT 2611, Australia \\ 9 Observatoire de Paris, PSL Research University, CNRS, Sorbonne Universités, UPMC, 61 Av. de l'Observatoire, 75014 Paris, \\ France
}

Received 16 February 2017 / Accepted 11 August 2017

\begin{abstract}
Context. The study of pre-main-sequence (PMS) stars with model-independent measurements of their masses is essential to check the validity of theoretical models of stellar evolution. The well-known PMS binary AB Dor A/C is an important benchmark for this task, since it displays intense and compact radio emission, which makes possible the application of high-precision astrometric techniques to this system.

Aims. We aim to revisit the dynamical masses of the components of $\mathrm{AB}$ Dor $\mathrm{A} / \mathrm{C}$ to refine earlier comparisons between the measurements of stellar parameters and the predictions of stellar models.

Methods. We observed in phase-reference mode the binary AB Dor A/C, 0.2" separation, with the Australian Long Baseline Array at $8.4 \mathrm{GHz}$. The astrometric information resulting from our observations was analyzed along with previously reported VLBI, optical (HIPPARCOS), and infrared measurements.

Results. The main star AB Dor A is clearly detected in all the VLBI observations, which allowed us to analyze the orbital motion of the system and to obtain model-independent dynamical masses of $0.90 \pm 0.08 M_{\odot}$ and $0.090 \pm 0.008 M_{\odot}$, for $\mathrm{AB}$ Dor A and $\mathrm{AB}$ Dor C, respectively. Comparisons with PMS stellar evolution models favor and age of 40-50 Myr for AB Dor A and of 25-120 Myr for AB Dor C.

Conclusions. We show that the orbital motion of the AB Dor A/C system is remarkably well determined, leading to precise estimates of the dynamical masses. Comparison of our results with the prediction of evolutionary models support the observational evidence that theoretical models tend to slightly underestimate the mass of the low-mass stars.
\end{abstract}

Key words. binaries: general - stars: pre-main sequence - radio continuum: stars - astrometry

\section{Introduction}

Precise astrometry of the orbital motion of stars in binary systems allows us to obtain accurate, model-independent estimates of the masses of the individual components, relevant to provide tests of stellar evolution models (Guirado et al. 1997; Torres et al. 2012; Reid \& Honma 2014). In the case of low- and very-lowmass stars, only a handful of stellar systems with dynamicallydetermined masses have been reported (Hillenbrand \& White 2004; Stassun et al. 2004; Mathieu et al. 2007). In this context, the pre-main-sequence (PMS) stellar system AB Doradus, the main star of the AB Doradus moving group (AB Dor-MG), is of particular interest. AB Doradus is placed at a distance of $\sim 15 \mathrm{pc}$ and it is actually a quadruple system formed by two pairs of stars separated by $9^{\prime \prime}, \mathrm{AB}$ Dor A/C and AB Dor Ba/Bb (Close et al. 2005; Guirado et al. 2006).

AB Dor A (=HD $36705=$ HIP 25647) is the main star of the system. Given its high-level of radio emission, during the early 90's AB Dor A was monitored astrometrically with VLBI as part of a program to link the optical reference frame, defined by the positions of the stars observed by the ESA's astrometric satellite HIPPARCos (Lindegren \& Kovalevsky 1995; Lestrade et al. 1995), and the celestial reference frame, defined by VLBI positions (Lestrade et al. 1995). The VLBI-HIPPARCOS combined data revealed the presence of a low-mass companion, $0.090 M_{\odot}$, AB Dor C (Guirado et al. 1997), orbiting AB Dor A at an average angular distance of $0.2^{\prime \prime}$. Imaging this new low-mass companion was difficult and it had to wait until the VLT near-infrared observations carried out by Close et al. $(2005,2007)$ using commissioning time of the Simultaneous Differential Imager (SDI) at the VLT, which allowed independent photometry of AB Dor C, free from contamination of the much brighter AB Dor A.

The combination of precise measurements of the dynamical mass and photometry of $\mathrm{AB}$ Dor $\mathrm{C}$ provided relevant consequences in terms of calibration of the mass-luminosity relationship for young, low-mass objects: basically, stellar model 
Table 1. Journal of observations of AB Dor A.

\begin{tabular}{cccrr}
\hline \hline Date (epoch) & Array configuration ${ }^{a}$ & UT range & $\begin{array}{r}\text { Beam size } \\
{[\mathrm{mas}]}\end{array}$ & $\begin{array}{r}\text { PA } \\
{\left[{ }^{\circ}\right]}\end{array}$ \\
\hline 11 Nov. 2007 (2007.863) & At, Cd, Hh, Ho, Mp, Pa & $10: 00-22: 00$ & $4.06 \times 1.84$ & -3.0 \\
25 Oct. 2010 (2010.816) & At, Cd, Ho, Mp, Pa & $11: 00-23: 00$ & $6.38 \times 5.38$ & -82.5 \\
16 Aug. 2013 (2013.625) & At, Cd, Hh, Ho, Mp, Pa, Ti, Ww & $15: 00-03: 00$ & $4.90 \times 2.35$ & -6.5 \\
\hline
\end{tabular}

Notes. ${ }^{(a)}$ At: Australia Telescope Compact Array, Cd: Ceduna, Hh: Hartebeesthoek, Ho: Hobart, Mp: Mopra, Pa: Parkes, Ti: DSS43 - NASA's Deep Space Network Tidbinbilla, Ww: Warkworth. Both RCP and LCP polarizations were recorded with a rate of 1024 Mbps (two polarizations, eight subbands per polarization, $16 \mathrm{MHz}$ per subband, 2 bits per sample), except at Hobart and Ceduna, with a recording rate of $512 \mathrm{Mbps}$ (two polarizations, four subbands per polarization, $8 \mathrm{MHz}$ per subband, 2 bits per sample).
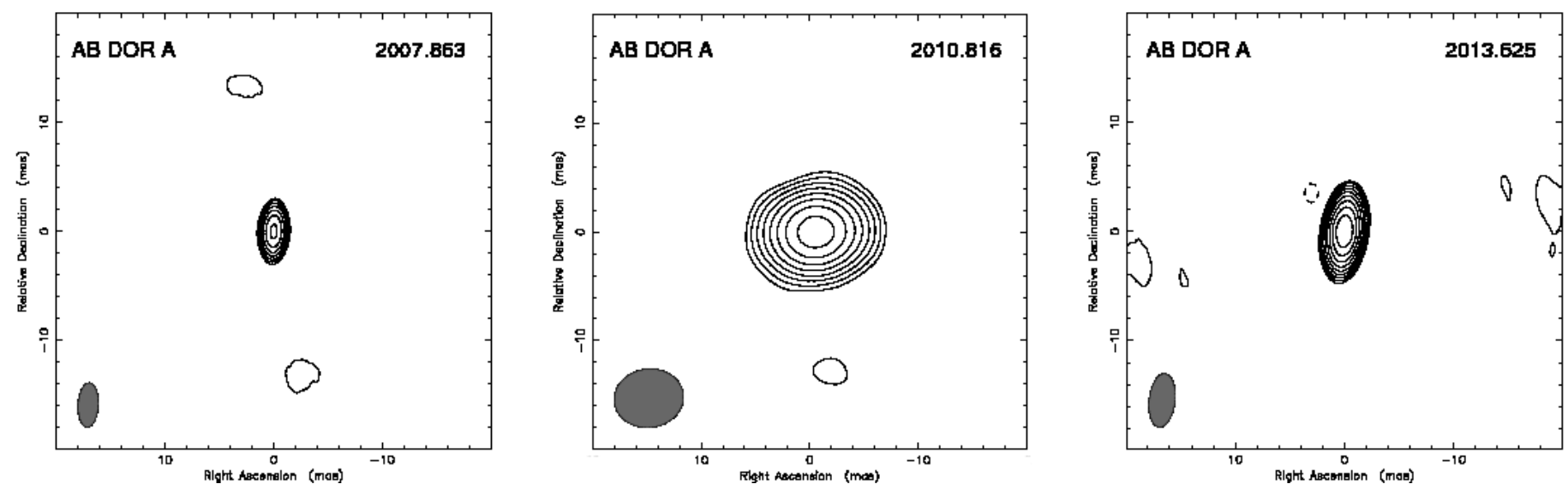

Fig. 1. Clean maps of the star AB Dor A at the three LBA epochs. In each map, the lowest contour level corresponds to three times the statistical root-mean-square $\left(0.20,0.05\right.$, and $\left.0.18 \mathrm{mJy} \mathrm{beam}^{-1}\right)$ with a scale factor between contiguous contours of $\sqrt{2}$. The peak flux densities in the images are, respectively, $11.1,5.84$, and $10.10 \mathrm{mJy}_{\text {beam }}{ }^{-1}$. For image parameters see Table 2 . In all the maps we have centered the position of AB Dor A at the origin. Absolute positions are presented in Table 3.

Table 2. Circular Gaussian fits corresponding to the VLBI maps of the components of AB Dor A.

\begin{tabular}{cccc}
\hline \hline Epoch & Component & $\begin{array}{c}\text { Flux } \\
(\mathrm{mJy})\end{array}$ & $\begin{array}{c}\text { Diameter } \\
\text { (mas) }\end{array}$ \\
\hline 2007.863 & A & $10.84 \pm 0.28$ & $2.97 \pm 0.05$ \\
2010.816 & A & $5.43 \pm 0.06$ & $4.81 \pm 0.04$ \\
2013.625 & A & $9.90 \pm 0.25$ & $2.56 \pm 0.04$ \\
\hline
\end{tabular}

predictions tend to underestimate the mass of the observed objects. Given the need for an accurate calibration of the models, any possible ambiguity or uncertainty affecting these few calibrating objects should be studied in detail. A vivid discussion about the nature, age, and characterization of this low-mass object followed from these results, including new observations made using the VLT chronograph (Boccaletti et al. 2008), VLT SINFONI (Thatte et al. 2007), Spitzer (Plavchan et al. 2009), and VLTI (Guirado et al. 2011).

In this work we present a deeper analysis of the VLBI observations of the AB Doradus system reported in Azulay et al. (2015), based on a new correlation pass centered on $\mathrm{AB}$ Dor A/C. Our analysis improves the determination of the orbital parameters of the system and obtains precise values of the dynamical masses of both components. We also discuss the comparisons of the dynamical masses of this pair with PMS stellar evolution models.

\section{Observations and data reduction}

AB Dor A was observed in three epochs using the Long Baseline Array (LBA), the Australian VLBI Network, at $8.4 \mathrm{GHz}$ (see Table 1). The observations were scheduled in phase-reference mode, using the ICRF-defining source BL Lac PKS 0516-621 (about $3.6^{\circ}$ away) as the phase calibrator. The sequence targetcalibrator-target lasted about four minutes. Results from these observations were reported in Azulay et al. (2015) for AB Dor B, the companion to AB Dor A, that is $10^{\prime \prime}$ away from it. Since both sources lie within the primary beam of all telescopes, the AB Dor A data were obtained from a new correlator pass using the nominal AB Dor A position as phase center.

The data calibration was carried out using the program Astronomical Image Processing System (AIPS) of the National Radio Astronomy Observatory (NRAO) with standard routines. We followed the process already described in Azulay et al (2015), and outlined briefly here: (i) calibration of the visibility amplitudes using the gain curves of each antenna and the system temperatures of each station; (ii) correction of both the parallactic angle and the ionospheric contribution; and (iii) fringe-search on the calibrator PKS 0516-621 to remove residual contributions to the phases, whose corrections were applied to the target source AB Dor A. The phase-referenced naturally-weighted images of $\mathrm{AB}$ Dor A were obtained using the Caltech imaging program DIFMAP (Shepherd et al. 1994) and are shown in Fig. 1. 
R. Azulay et al.: The AB Doradus system revisited: The dynamical mass of AB Dor A/C

Table 3. Compilation of astrometric measurements for the AB Dor A system.

\begin{tabular}{|c|c|c|c|c|}
\hline \multicolumn{5}{|c|}{ Relative positions $\mathrm{AB}$ Dor $\mathrm{A}-\mathrm{AB}$ Dor $\mathrm{C}$} \\
\hline Epoch & Instrument & $\Delta \alpha(\operatorname{arcsec})$ & $\Delta \delta(\operatorname{arcsec})$ & Reference \\
\hline 2004.093 & VLT/NACO (IR) & $-0.125 \pm 0.008$ & $0.094 \pm 0.006$ & (1) \\
\hline 2004.825 & " & $-0.106 \pm 0.005$ & $0.191 \pm 0.005$ & (2) \\
\hline 2004.877 & $\prime \prime$ & $-0.106 \pm 0.005$ & $0.192 \pm 0.005$ & (2) \\
\hline 2005.017 & " & $-0.089 \pm 0.004$ & $0.200 \pm 0.007$ & (3) \\
\hline 2006.066 & $" 1$ & $0.003 \pm 0.003$ & $0.202 \pm 0.003$ & (3) \\
\hline \multicolumn{5}{|c|}{ Absolute positions AB Dor A } \\
\hline 1990.3888 & HIPPARCOS & $52844.77474 \pm 0.00026$ & $-652656.24163 \pm 0.00074$ & (4) \\
\hline 1990.5640 & " & $52844.78652 \pm 0.00025$ & $-652656.22719 \pm 0.00068$ & (4) \\
\hline 1991.0490 & " & $52844.77578 \pm 0.00024$ & $-652656.26151 \pm 0.00075$ & (4) \\
\hline 1991.5330 & $\prime \prime$ & $52844.78942 \pm 0.00025$ & $-652656.07567 \pm 0.00083$ & (4) \\
\hline 1992.0180 & " & $52844.78202 \pm 0.00024$ & $-652656.11601 \pm 0.00088$ & (4) \\
\hline 1992.2329 & VLBI & $52844.77687 \pm 0.00019$ & $-652656.00487 \pm 0.00070$ & (4) \\
\hline 1992.6849 & $" \prime$ & $52844.80124 \pm 0.00018$ & $-652655.93947 \pm 0.00063$ & (4) \\
\hline 1993.1233 & " & $52844.78492 \pm 0.00024$ & $-652655.91371 \pm 0.00084$ & (4) \\
\hline 1994.8137 & $\prime \prime$ & $52844.81768 \pm 0.00019$ & $-652655.68661 \pm 0.00047$ & (4) \\
\hline 1995.1425 & " & $52844.80247 \pm 0.00027$ & $-652655.62477 \pm 0.00114$ & (4) \\
\hline 1996.1507 & $\prime \prime$ & $52844.81137 \pm 0.00013$ & $-652655.48519 \pm 0.00032$ & (4) \\
\hline 1996.3607 & " & $52844.81776 \pm 0.00018$ & $-652655.37851 \pm 0.00104$ & (4) \\
\hline 2007.863 & $\prime \prime$ & $52844.91581 \pm 0.00018$ & $-652653.86529 \pm 0.00104$ & (5) \\
\hline 2010.816 & $\prime \prime$ & $52844.94276 \pm 0.00018$ & $-652653.45468 \pm 0.00104$ & (5) \\
\hline 2013.625 & " & $52844.96397 \pm 0.00018$ & $-652652.99578 \pm 0.00104$ & (5) \\
\hline
\end{tabular}

Notes. The standard deviation of the relative position corresponds to the uncertainty based on the signal-to-noise ratio of the peaks of brightness of $\mathrm{AB}$ Dor $\mathrm{Ba}$ and $\mathrm{Bb}$. The absolute positions were obtained with reference to the IERS coordinate of the external quasar PKS 0516-621 $(\alpha=$ $5^{\mathrm{h}} 16^{\mathrm{m}} 44^{\mathrm{s}} .926178, \delta=-62^{\circ} 7^{\prime} 5^{\prime \prime} \cdot 38930$ ). The standard deviation of the absolute position includes, in addition to the uncertainty of their respective peaks of brightness, the contribution of the propagation media and the reference source structure.

References. (1) Close et al. (2005); (2) Nielsen et al. (2005); (3) Close et al. (2007); (4) Guirado et al. (1997); (5) This study.

Table 4. Estimates of the astrometric and orbital parameters of AB Dor A.

\begin{tabular}{rrc}
\hline \hline Parameter & \multicolumn{1}{c}{ Value } & Range explored by the MCMC \\
\hline$\alpha_{0}(\mathrm{~h} \mathrm{~m} \mathrm{~s})$ & $52844.79483 \pm 0.00025$ & $-0.01-0.01$ \\
$\delta_{0}\left({ }^{\circ}{ }^{\prime \prime}\right)$ & $-652655.91774 \pm 0.00214$ & $-0.15-0.15$ \\
$\mu_{\alpha}\left(\mathrm{s} \mathrm{yr}^{-1}\right)$ & $0.00755 \pm 0.00001$ & $0.00-0.01$ \\
$\mu_{\delta}\left({\left.\operatorname{arcsec~} \mathrm{yr}^{-1}\right)}_{\pi(\mathrm{arcsec})}\right.$ & $0.14101 \pm 0.00006$ & $0.135-0.145$ \\
$P(\mathrm{yr})$ & $0.0664 \pm 0.0003$ & $0.025-0.075$ \\
$a_{\mathrm{A}}\left(^{\prime \prime}\right)$ & $11.78 \pm 0.10$ & $0-30$ \\
$m_{\mathrm{C}} / m_{\mathrm{A}}$ & $0.0314 \pm 0.0010$ & $0.0-0.2$ \\
$e$ & $0.101 \pm 0.003$ & $0-1$ \\
$i\left(^{\circ}\right)$ & $0.59 \pm 0.05$ & $0-1$ \\
$\omega_{A}\left({ }^{\circ}\right)$ & $65 \pm 1$ & $0-90$ \\
$\Omega\left(^{\circ}\right)$ & $114 \pm 5$ & $-180-180$ \\
$T_{0}$ & $1991.9 \pm 0.2$ & $0-180$ \\
$m_{\mathrm{A}}\left(M_{\odot}\right)$ & $0.89 \pm 0.08$ & \\
$m_{\mathrm{C}}\left(M_{\odot}\right)$ & $0.090 \pm 0.008$ & \\
\hline
\end{tabular}

Notes. Orbital parameters correspond to the least-square fit analysis, which are coincident within uncertainties, with those provided by a Bayesian approach based on MCMC. For the latter method, the range of explored parameters is also shown. We notice that we have conservatively increased the uncertainties of each parameter to be the largest one resulting from both approaches. The reference epoch is 2000.0. See Sect. 3.2. for details.

\section{Results}

\subsection{Maps of the $A B D o r A / C$ system}

The star AB Dor A is clearly detected in all three maps presented in Fig. 1. Although AB Dor A is known to be a strong radio emitter (White et al. 1988) and other VLBI observations have been previously reported (Guirado et al. 1997), these are (to our knowledge) the first VLBI images published of this object. To model these VLBI images, we made least-squares fits to the interferometric visibilities by using circular Gaussians (i.e., model fitting in the $u v$-plane; see Table 2). We notice that the use of 


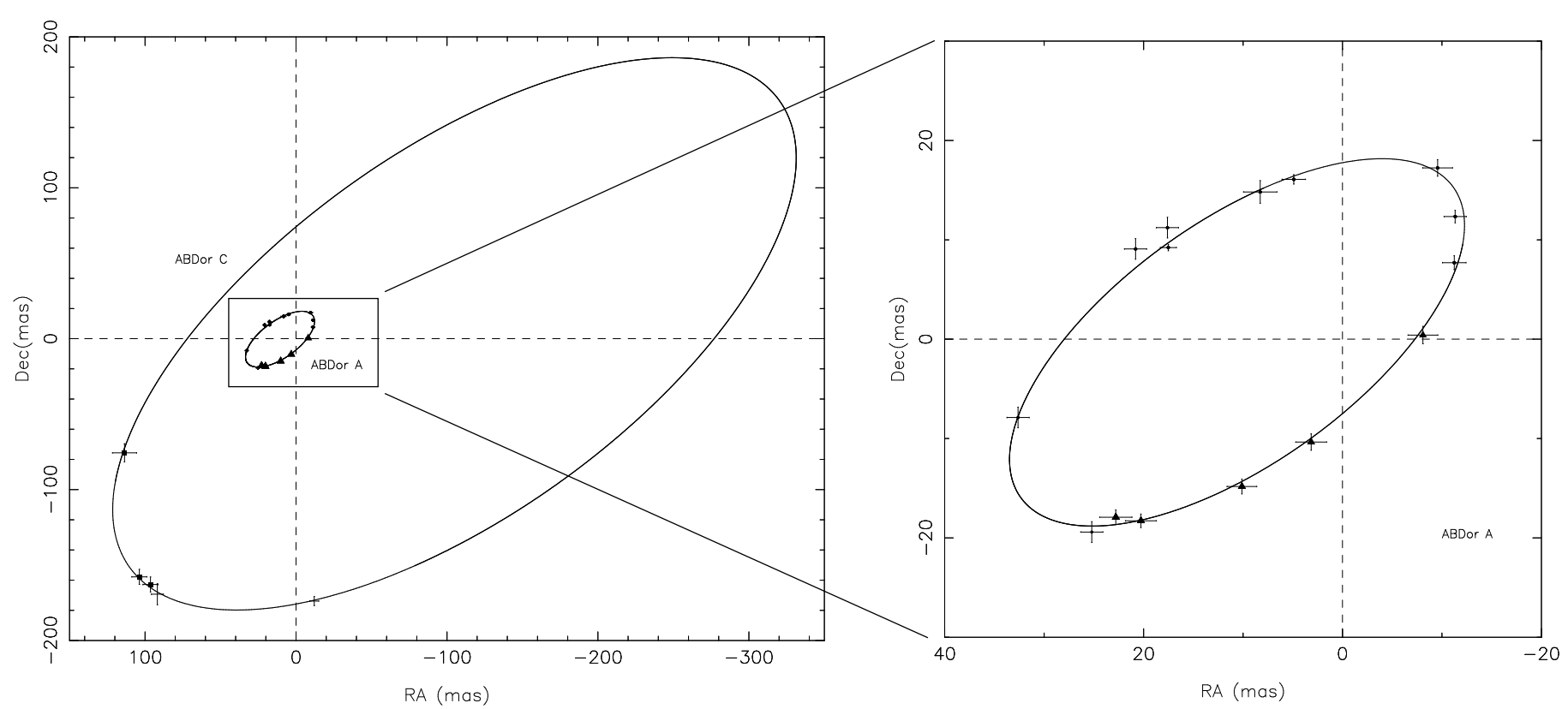

Fig. 2. Absolute orbits of the components $\mathrm{AB}$ Dor $\mathrm{A}$ and $\mathrm{AB}$ Dor $\mathrm{C}$ using the orbital elements in Table 4 (with $a_{\mathrm{A}}$ and $a_{\mathrm{C}}$, respectively). Positions of the component A (triangles, HIPPARCos data; circles, VLBI data) and C (squares) are marked. The center of mass of the system is placed at the origin.
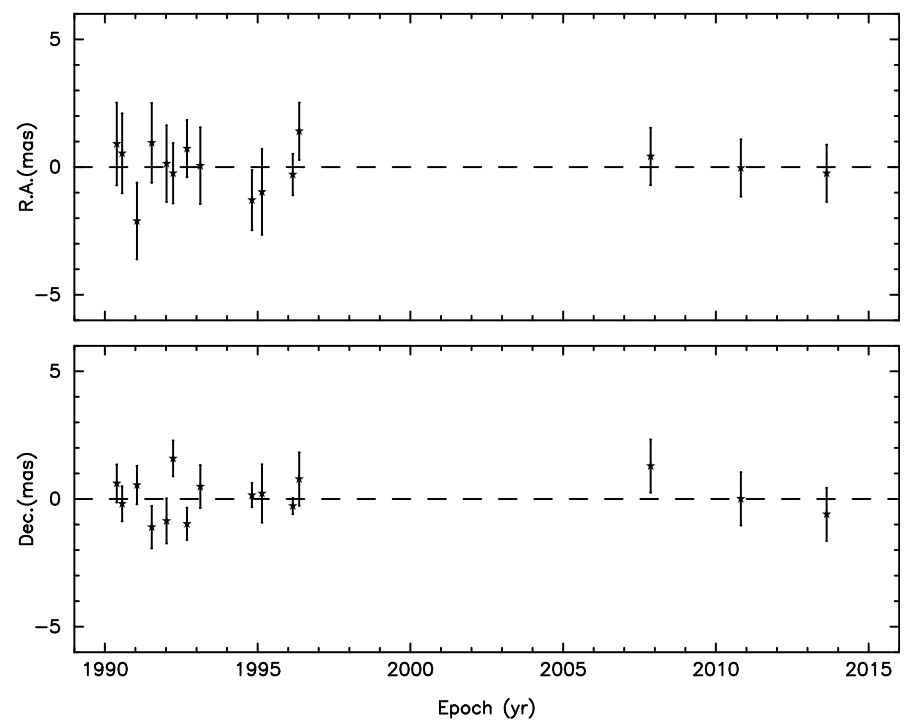

Fig. 3. Postfit residuals in right ascension (upper panel) and declination (lower panel) of the component $\mathrm{AB}$ Dor $\mathrm{A}$. The weighted rms is 1.1 mas (see text).

elliptical Gaussians is not necessary to model the structure of $\mathrm{AB}$ Dor A, adding unnecessary free parameters to the analysis.

From the flux density displayed in Table 2 and the parallax given in Table 4, we calculated a mean value of the absolute radio luminosity for AB Dor A of $L_{\mathrm{R}}=3.16 \times 10^{15} \mathrm{erg} \mathrm{Hz}^{-1} \mathrm{~s}^{-1}$, very similar to other radio stars of the AB Dor-MG. This value indicates that the radio emission from this star arises from non-thermal, mildly relativistic electrons, or non-thermal gyrosynchtron emission.

The companion $\mathrm{AB}$ Dor $\mathrm{C}$, directly imaged by Close et al. (2005) with the NACO SDI camera, was not detected in any of the three epochs, with a noise floor of $0.20,0.05$, and $0.18 \mathrm{mJy}$, respectively. We made a specific search for the emission of
AB Dor $C$ by stacking the individual images of the system. Taking advantage of the predicted position of $\mathrm{AB}$ Dor $\mathrm{C}$ with respect to AB Dor A at all observing epochs (Guirado et al. 2006; see Sect. 3.2), we shifted each of the images so that the expected position of component $\mathrm{C}$ was at the origin of coordinates. In practice, we made a stacked image by convolving the three images with a common restoring beam $\left(6.4 \times 5.4\right.$ mas with a PA of $-90^{\circ}$, which is nearly that of epoch 2010.816 , the largest beam given in Table 1). Although there was no detection of $\mathrm{AB}$ Dor $\mathrm{C}$, we set, thus, an upper bound to the radio emission of this object of $0.07 \mathrm{mJy}$.

\subsection{Orbital parameters of $A B D$ or $A / C$}

The new detections of AB Dor A in our VLBI observations have allowed us to determine new absolute positions of the star (i.e., positions of the star with respect to the ICRF defining source the PKS 0516-621), which are shown in Table 3 along with other absolute and relative positions (AB Dor A - AB Dor C) previously reported. Our new VLBI positions of AB Dor A necessarily brought us to a re-calculation of the orbital elements and masses of this system.

To carry out this revision, we used two fitting methods, a least-squares analysis and a Bayesian approach, that we describe in turn. First, we followed the least-squares fit method detailed in Azulay et al. (2015). We combined the absolute positions of $\mathrm{AB}$ Dor $\mathrm{A}$ and all the relative positions available to determine the astrometric and orbital parameters which are shown in Table 4. As stated in Azulay et al. (2015), a priori values of the orbital elements are essential to find convergence of the least-squares method; we used the orbital elements given in Guirado et al. (2006) as a priori values for the least-squares analysis, which greatly facilitated the convergence of our procedure. Diagrams of the absolute orbits are shown in Fig. 2. The weighted rms of the postfit residuals of AB Dor A (plotted in Fig. 3) is 1.1 mas; although some unmodelled effects are present, the shown uncertainties are sufficient to exclude the presence of 


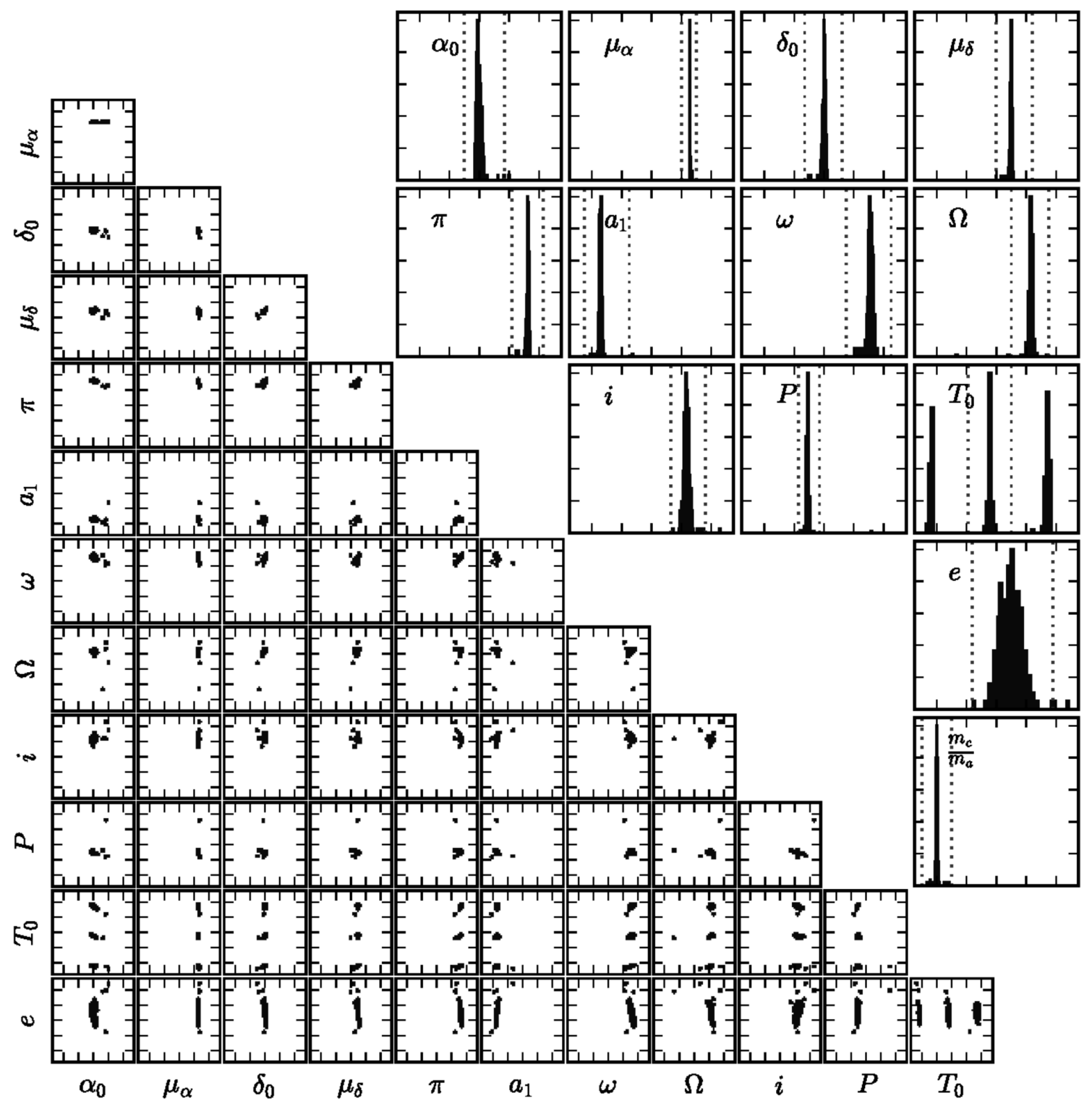

Fig. 4. Results of our MCMC exploration of the orbital-parameter space. All axis ranges are normalized to the values given in Table 4. Bottom left: cross-correlations between parameters. Top right: posterior probability distributions. Dotted lines mark the ranges used to derive the averages (and standard deviations) of the parameters, given in Table 4 . We note the secondary peak in $T_{0}$, which is separated from the first peak by the orbital period.

further companions to this system with masses below $0.15 M_{\mathrm{J}}$ at a distance of $1 \mathrm{AU}$ from the central star.

In complement to the above approach, we used a Bayesian approach based on a Markov-chain Monte Carlo (MCMC) routine, which in turn uses the Metropolis-Hastings algorithm (Robert 2015). We explored the solution space corresponding to the range of parameters given in Table 4 . The orbital parameters obtained agree within uncertainties with those resulting from the least-squares method, which are already reported in Table 4 . We show in Fig. 4 the posterior probability distributions for all parameters, together with their cross-correlations. The coincidence of both methods shows the robustness of our orbital solution for the $\mathrm{AB}$ Dor $\mathrm{A} / \mathrm{C}$ system.

From the values of the mass ratio $m_{\mathrm{C}} / m_{\mathrm{A}}$, the period $P$ (in years), and the parallax $\pi$ (in arcsec) in Table 4 , we could derive the sum of the masses of the system using the Kepler's third law $m_{\mathrm{A}}+m_{\mathrm{C}}=\left[1+\left(\frac{m_{\mathrm{C}}}{m_{\mathrm{A}}}\right)^{-1}\right]^{3} \frac{\left(a_{\mathrm{A}} / \pi\right)^{3}}{P^{2}}$,

which is $0.98 \pm 0.08 M_{\odot}$. Moreover, with the semimajor axis of the absolute orbit of AB Dor A, $a_{\mathrm{A}}$, and using the Kepler's third law in the form

$$
\frac{m_{\mathrm{C}}^{3}}{\left(m_{\mathrm{A}}+m_{\mathrm{C}}\right)^{2}}=\frac{\left(a_{\mathrm{A}} / \pi\right)^{3}}{P^{2}},
$$

we derived a value of $0.090 \pm 0.008 M_{\odot}$ for the mass of $\mathrm{AB}$ Dor C. Since we did not detect the component AB Dor C in our VLBI observations, we could not determine the semimajor axis of the absolute orbit of this component, $a_{\mathrm{C}}$ and, consequently, the mass of the component $\mathrm{A}$; so the mass of AB Dor A follows from subtracting the value of the sum of the mass of both 

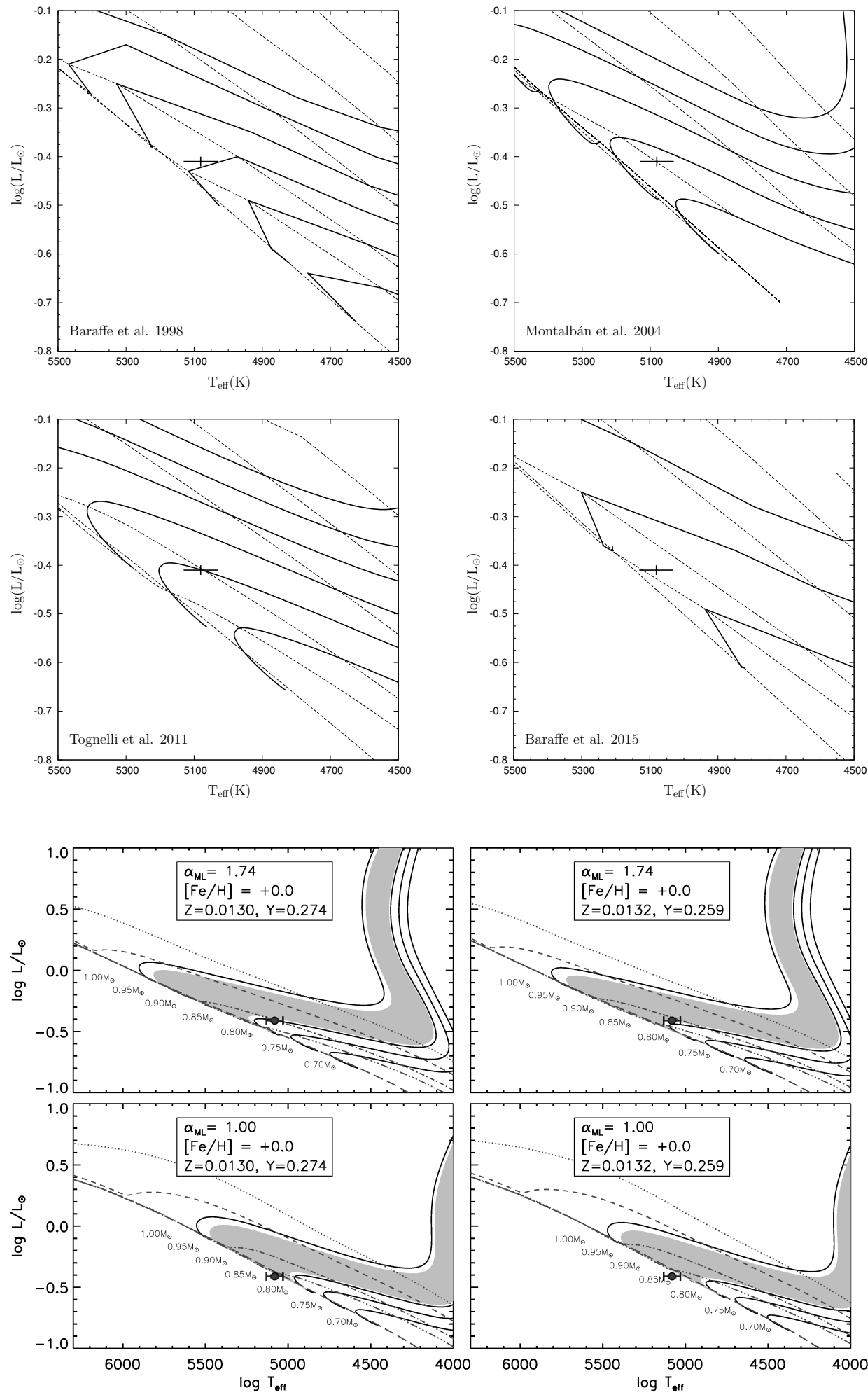

Fig. 5. Comparison of $A B$ Dor A with some PMS theoretical models (BCAH98, top left; MDKH04, top right; TDP12, bottom left; BHAC15, bottom right). Isochrones (dashed lines) correspond to 10 (top isochrone), 16, 25, 40, 50, and $100 \mathrm{Myr}$. Isomasses (solid lines) are for 0.75 (bottom isomass), $0.80,0.85,0.90,0.95$, and $1.00 M_{\odot}$ in the case of BCAH98 and TDP12, for 0.80 (bottom isomass), 0.85 , $0.90,0.95$, and $1.00 M_{\odot}$ in the case of MDKH04, and for 0.80 (bottom isomass), 0.90 , and $1.00 M_{\odot}$ in the case of BHAC 15 . We have considered the value $\alpha_{\mathrm{ML}}=1.90$ for BCAH98 and the value $\alpha_{\mathrm{ML}}=1.74$ for TDP12.

Fig. 6. Comparison of $A B$ Dor $A$ with a set of PMS evolutionary tracks with $[\mathrm{Fe} / \mathrm{H}]=$ 0 for different initial helium abundances (standard abundance, left panels; low abundance, right panels), and for different mixing length values $\alpha_{\mathrm{ML}}(1.74$, top panels; 1.00 , bottom panels). The shaded area corresponds to the region between track with masses in $0.82-0.98 M_{\odot}$, as given by the uncertainty on dynamical mass. components and the value of the mass of $\mathrm{AB}$ Dor $\mathrm{C}$. We obtained $m_{\mathrm{A}}=0.89 \pm 0.08 M_{\odot}$.

\section{Discussion}

The dynamical determination of the individual masses of $\mathrm{AB}$ Dor $\mathrm{A}$ and $\mathrm{AB}$ Dor $\mathrm{C}$ is an important result that can be used to test theoretical predicitons of PMS stellar evolution models. Both $\mathrm{AB}$ Dor $\mathrm{A}$ and $\mathrm{C}$ can be placed in $\mathrm{H}-\mathrm{R}$ diagrams using the measured luminosity and the effective temperature, along with theoretical isomasses and isochrones. Different theoretical models are used depending on their availability according to the masses of the objects.

Regarding AB Dor A, in Fig. 5 we compared the measurements of the effective temperature and luminosity $(5081 \pm 50 \mathrm{~K}$ and $0.388 \pm 0.008 L_{\odot}$, respectively; Close et al. 2007) with the predictions of the PMS models of Baraffe et al. (1998; BCAH98), Montalbán et al. (2004; MDKH04), Tognelli et al. (2011, 2012; TDP12), and Baraffe et al. (2015; BHAC15). We refer to the cited papers for a complete discussion of the 

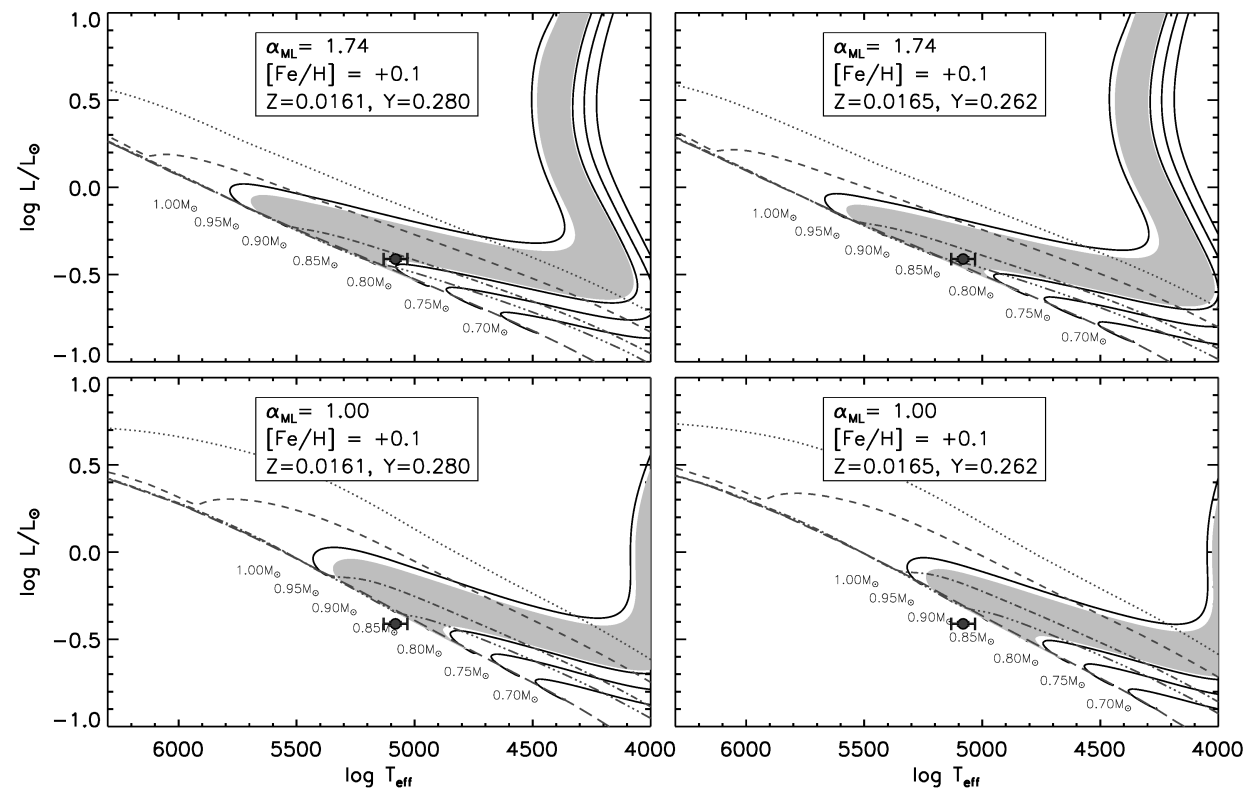

Fig. 7. As Fig. 6 for $[\mathrm{Fe} / \mathrm{H}]=+0.1$.
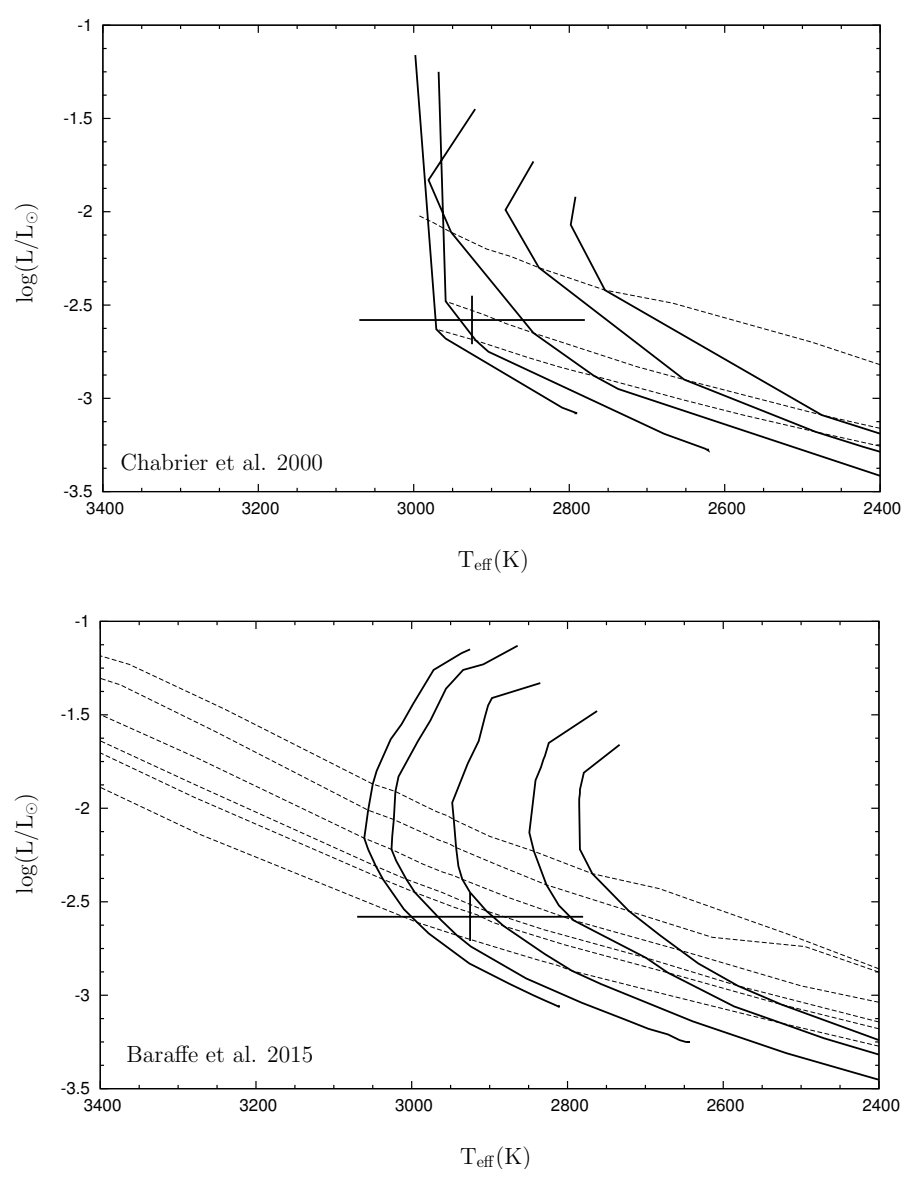

Fig. 8. Comparison of $\mathrm{AB}$ Dor $\mathrm{C}$ with $\mathrm{PMS}$ theoretical models of Chabrier et al. (2000) (top) and Baraffe et al. (2015) (bottom). Isochrones (dashed lines) correspond to 10 (top isochrone), 50, and $100 \mathrm{Myr}$, in the case of the DUSTY models, and to 10 (top isochrone), $16,25,40,50$, and $100 \mathrm{Myr}$, in the case of BHAC15. Isomasses (solid lines), in both cases, are for 0.04 (rightmost isomass), 0.05, 0.07, 0.09, and $0.10 M_{\odot}$.

physics and calculations involved. The two first model comparisons (BCAH98 and MDKH04) are reported in Guirado et al. (2011) and are shown here again for completeness along with the other, newer models. Considering the isomasses displayed in Fig. 5, we see a general good agreement between the predicted and the dynamical mass, although a persistent underprediction is seen at all models (between $2 \%$ and 6\%). Nevertheless, these differences are covered by the uncertainties shown for the measurements of effective temperature and luminosity. Regarding the isochrones, the models shown in Fig. 5 favor an age for $\mathrm{AB}$ Dor A of 40-50 Myr, very similar to that predicted in Guirado et al. (2011).

We took advantage of the flexibility of the TDP12 models to investigate how theoretical predictions are affected by the adopted values of $Y$ (initial helium mass fractional abundance) and $Z$ (initial total metallicity) by showing the effect of varying these parameters on the tracks position in the H-R diagram. The quantities $Y$ and $Z$ are not directly obtained from the observations, but they are derived from the measured $[\mathrm{Fe} / \mathrm{H}]$. Therefore, we computed models for $[\mathrm{Fe} / \mathrm{H}]=+0.0$ and +0.1 , the extreme values of the reported estimates of $[\mathrm{Fe} / \mathrm{H}]$ for the $\mathrm{AB}$ Dor-MG, namely, $[\mathrm{Fe} / \mathrm{H}]=0.02 \pm 0.02$ (Barenfeld et al. 2013), $0.10 \pm 0.03$ (Biazzo et al. 2012), and $0.04 \pm 0.05$ (Viana Almeida et al. 2009). We analyzed the effect of the models of adopting different initial helium content in the star, by producing a set of stellar tracks with a low initial helium abundance. The results of the computations with the two $[\mathrm{Fe} / \mathrm{H}]$ and $Y$ values are plotted in Figs. 6 and 7, for stellar tracks in the mass range $0.70-0.95 M_{\odot}$. For each figure, models with two values of the mixing length parameter, namely $\alpha_{\mathrm{ML}}=1.74$ (solar calibrated mixing length parameter, top panels) and $\alpha_{\mathrm{ML}}=1.00$ (bottom panel) are shown. We overplotted the $16,25,40,50$, and $100 \mathrm{Myr}$ isochrones.

By comparing the models shown in panels of Figs. 6 and 7 it emerges that the variation of $[\mathrm{Fe} / \mathrm{H}]$ produces a relevant effect on theoretical models. Indeed, an increase of $0.1 \mathrm{dex}$ in $[\mathrm{Fe} / \mathrm{H}]$ produces an increase of about $20 \%$ in $Z$, which significantly alters the position of the tracks in the HR-diagram making the stars cooler and fainter. Consequently, the derived mass gets larger if $[\mathrm{Fe} / \mathrm{H}]=+0.1$ models are adopted. Moreover, at a fixed $[\mathrm{Fe} / \mathrm{H}]$, a reduction of the initial helium content produces cooler and fainter stars. Even in this case the derived mass slightly increases, partially reducing the discrepancy between the measured and inferred stellar mass. Concerning the mixing length parameter, from the comparisons it seems to emerge that TDP12 

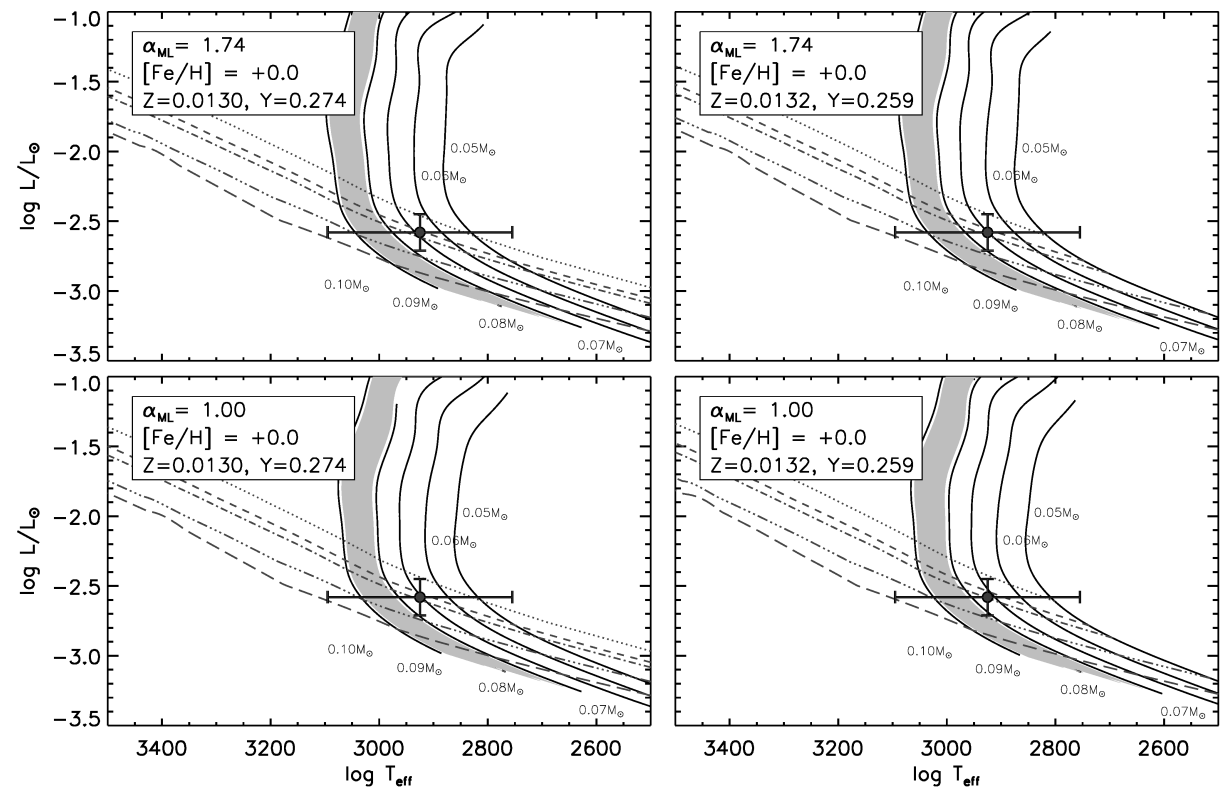

Fig. 9. Comparison of $\mathrm{AB}$ Dor $\mathrm{C}$ with a set of PMS evolutionary tracks with $[\mathrm{Fe} / \mathrm{H}]=$ 0 for different initial helium abundances (standard abundance, left panels; low abundance, right panels), and for different mixing length values $\alpha_{\mathrm{ML}}(1.74$, top panels; 1.00 , bottom panels). The shaded area corresponds to the region between track with masses in $0.082-0.099 M_{\odot}$, as given by the uncertainty on dynamical mass.
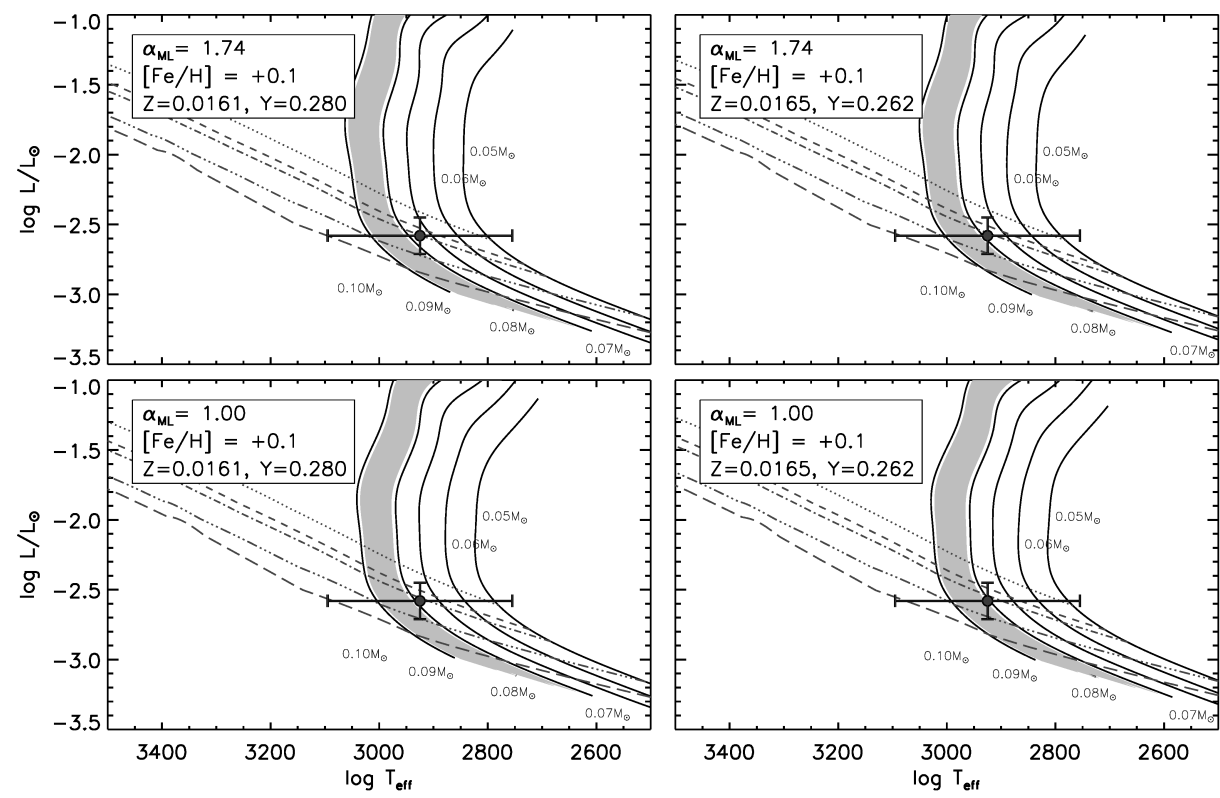

Fig. 10. As Fig. 9 for $[\mathrm{Fe} / \mathrm{H}]=+0.1$.

solar calibrated models, i.e., $\alpha_{\mathrm{ML}}=1.74$ are probably preferred (similarly to what found by Guirado et al. 2011). However, we remark that the uncertainties on both the luminosity and temperature do not allow us to reject the $\alpha_{\mathrm{ML}}=1.00$ models.

From the comparisons shown in Figs. 6 and 7, we can conclude that $\mathrm{AB}$ Dor $\mathrm{A}$ is better reproduced by the set of values $[\mathrm{Fe} / \mathrm{H}]=+0.1$ and $\alpha_{\mathrm{ML}}=1.74$, with a derived mass of $\sim 0.85 \mathrm{M}_{\odot}$ and an age of about 40-50 Myr. However, more precise measurements of the total luminosity and the effective temperature are required to better constrain the models.

Regarding $\mathrm{AB}$ Dor $\mathrm{C}$, the mass derived for this object $\left(0.090 \pm 0.008 M_{\odot}\right)$ does not differ substantially from that reported in Guirado et al. (2006), therefore the comparisons reported in Close et al. (2007) and Boccaletti et al. (2008) still hold. For completeness we include here the H-R diagram (Fig. 8) using the recent BHAC15 models (along with those from Chabrier et al. 2000; DUSTY models). We used the effective temperature from Close et al. (2007) and the luminosity derived using the $K_{\mathrm{s}}$ magnitude given by Boccaletti et al. (2008) and derived using the procedure (distance and bolometric correction) given in Close et al. (2007). The models predict a compatible estimate with our dynamical mass, although slightly underestimating the result (by approximately 10\%; although this comparison is limited by the uncertainties in the effective temperature). Both models suggest an age between 25 and $120 \mathrm{Myr}$, consistent with the values published in Close et al. (2007) and Boccaletti et al. (2008).

We performed the analysis using also the TDP12 models. Similarly to what done for the AB Dor A component, we compared the data with models computed with the two values of $[\mathrm{Fe} / \mathrm{H}]$, namely +0.0 and +0.1 , with the standard and low initial helium content. Even in this case we used models with two mixing length parameter values, namely 1.74 and 1.00 . The results are shown in Figs. 9 and 10, were we plotted the tracks in the $0.05-0.10 M_{\odot}$ mass range and the isochrones of $25,40,50,100$ and 200 Myr. To place the star in the H-R diagram, we also used 
the effective temperature given by Close et al. (2007) and the luminosity obtained from the $K_{\mathrm{s}}$ magnitude given by Boccaletti et al. (2008).

As clearly visible, the models are fully compatible, within the observational uncertainties, with the data. In this case, the tracks are only marginally affected by the adopted chemical composition and mixing length parameter. Consequently, given the relatively large uncertainties, we can not state which of the plotted sets gets the best agreement. From the comparisons, we derived a central mass of about $0.07 M_{\odot}$ and a central age of 40-50 Myr. We also tried to compare the TDP12 models with the data using the luminosity given by Close et al. (2007), which is about 0.1 dex lower than that derived using the Boccaletti et al. (2008) $K_{\mathrm{s}}$ magnitude. In this case, we obtained a mass of approximately $0.08 M_{\odot}$ and an age of about $80 \mathrm{Myr}$.

\section{Conclusions}

We revisited the $8.4 \mathrm{GHz}$ observations presented in Azulay et al. (2015) by re-correlating the observed data centered in the PMS stellar system $\mathrm{AB}$ Dor $\mathrm{A} / \mathrm{C}$, thus complementing the previous astronomic monitoring by Guirado et al. (1997, 2006). The main star AB Dor A was clearly detected in all three epochs, with a flux density varying between 5 and $11 \mathrm{mJy}$, meanwhile the lowmass component $\mathrm{AB}$ Dor $\mathrm{C}$ was not detected. We have set an upper bound to the radio emission of this low-mass object of $0.07 \mathrm{mJy}$.

With these new epochs of observations of AB Dor A, we reanalyzed the orbital motion of the system following two different approaches (based on least-squares fits and MCMC analysis, respectively) which combined absolute positions of $\mathrm{AB}$ Dor A (from VLBI and HIPPARCOS) and near-infrared relative positions available (from VLT/NACO). From the estimated orbital parameters we determined values of the masses of $0.89 \pm 0.08 M_{\odot}$ and $0.090 \pm 0.008 M_{\odot}$ for $\mathrm{AB}$ Dor A and AB Dor C, respectively.

We compared these dynamical masses with the predictions of different PMS stellar evolution models. In general, we found a good agreement between the predicted and the dynamical masses, although a slight underestimation (already present in previous works) is predicted by all models. This data-theory comparison is limited by the uncertainties in the determination of the effective temperature and/or the luminosity. We have shown that the theoretical predictions could also be affected by the adopted chemical composition, that with a small variation may cause large differences in the derived masses.

Regarding the age, the models considered in this paper favor an age of 40-50 Myr for AB Dor A and 25-120 Myr for $\mathrm{AB}$ Dor $\mathrm{C}$. This model-dependent age is on the younger side of the age estimates of several authors: 40-50 Myr (Zuckerman et al. 2004; López-Santiago et al. 2006; Guirado et al. 2011), 50-100 Myr (Nielsen et al. 2005; Janson et al. 2007; Boccaletti et al. 2008). However, it is barely coincident with (especially in the case of AB Dor A) the lower limit of the AB Dor nucleus star (110 Myr) established by Barenfeld et al. (2013) or the recent isochronical age of the AB Dor moving group (130-200 Myr, Bell et al. 2015).
New, more sensitive VLBI observations will be needed to detect the companion AB Dor C. Although the orbital motion of the $\mathrm{AB}$ Doradus system is remarkable well determined, knowledge of the submas-precise radio position of $\mathrm{AB}$ Dor $\mathrm{C}$ will dramatically improve the precision of the estimated dynamical masses.

Acknowledgements. R.A., J.C.G., J.M.M, and E.R. were partially supported by the Spanish MINECO projects AYA2012-38491-C02-01 and AYA2015-63939C2-2-P and by the Generalitat Valenciana project PROMETEO/2009/104 and PROMETEOII/2014/057. E.T. was supported by the "PRA 2016 Università di Pisa". E.T. also acknowledges the INFN iniziativa specifica TAsP. The Long Baseline Array is part of the Australia Telescope National Facility which is funded by the Commonwealth of Australia for operation as a National Facility managed by CSIRO.

\section{References}

Azulay, R., Guirado, J. C., Marcaide, J. M., Martí-Vidal, I., \& Arroyo-Torres, B. 2014, A\&A, 561, A38

Azulay, R., Guirado, J. C., Marcaide, J. M., et al. 2015, A\&A, 578, A16 Baraffe, I., Chabrier, G., Allard, F., \& Hauschildt, P. H. 1998, A\&A, 337, 403 Baraffe, I., Homeier, D., Allard, F., \& Chabrier, G. 2015, A\&A, 577, A42 Barenfeld, S. A., Bubar, E. J., Mamajek, E. E., \& Young, P. A. 2013, ApJ, 766, 6

Bell, C. P. M., Mamajek, E. E., \& Naylor, T. 2015, MNRAS, 454, 593 Biazzo, K., D’Orazi, V., Desidera, S., et al. 2012, MNRAS, 427, 2905 Boccaletti, A., Chauvin, G., Baudoz, P., \& Beuzit, J.-L. 2008, A\&A, 482, 939 Chabrier, G., Baraffe, I., Allard, F., \& Hauschildt, P. 2000, ApJ, 542, 464 Close, L. M., Lenzen, R., Guirado, J. C., et al. 2005, Nature, 433, 286 Close, L. M., Thatte, N., Nielsen, E. L., et al. 2007, ApJ, 665, 736 Guirado, J. C., Reynolds, J. E., Lestrade, J.-F., et al. 1997, ApJ, 490, 835 Guirado, J. C., Martí-Vidal, I., Marcaide, J. M., et al. 2006, A\&A, 446, 733 Guirado, J. C., Marcaide, J. M., Martí-Vidal, I., et al. 2011, A\&A, 533, A106 Hillenbrand, L. A., \& White, R. J. 2004, ApJ, 604, 741

Janson, M., Brandner, W., Lenzen, R., et al. 2007, A\&A, 462, 615 Lestrade, J.-F., Jones, D. L., Preston, R. A., et al. 1995, A\&A, 304, 182 Lindegren, L., \& Kovalevsky, J. 1995, A\&A, 304, 189

López-Santiago, J., Montes, D., Crespo-Chacón, I., \& Fernández-Figueroa, M. J. 2006, ApJ, 643, 1160

Martí-Vidal, I., Marcaide, J. M., Quirrenbach, A., et al. 2011, A\&A, 529, A115 Mathieu, R. D., Baraffe, I., Simon, M., Stassun, K. G., \& White, R. 2007, Protostars and Planets V, 411, eds. B. Reipurth, D. Jewitt, \& K. Keil (Tucson: University of Arizona Press)

Mérand, A., Bordé, P., \& Coudé du Foresto, V. 2006, A\&A, 447, 783

Montalbán, J., D’Antona, F., Kupka, F., \& Heiter, U. 2004, A\&A, 416, 1081

Nielsen, E. L., Close, L. M., Guirado, J. C., et al. 2005, Astron. Nachr., 326, 1033

Plavchan, P., Werner, M. W., Chen, C. H., et al. 2009, ApJ, 698, 1068

Reid, M. J., \& Honma, M. 2014, ARA\&A, 52, 339

Robert, C. P. 2015, ArXiv e-prints [arXiv: 1504.018963]

Shepherd, M. C., Pearson, T. J., \& Taylor, G. B. 1994, BAAS, 26, 987

Stassun, K. G., Mathieu, R. D., Vaz, L. P. R., Stroud, N., \& Vrba, F. J. 2004, ApJS, 151, 357

Tatulli, E., Millour, F., Chelli, A., et al. 2007, A\&A, 464, 29

Thatte, N., Abuter, R., Tecza, M., et al. 2007, MNRAS, 378, 1229

Tognelli, E., Prada Moroni, P. G., \& Degl'Innocenti, S. 2011, A\&A, 533, A109

Tognelli, E., Degl'Innocenti, S., \& Prada Moroni, P. G. 2012, A\&A, 548, A41

Torres, R. M., Loinard, L., Mioduszewski, A. J., et al. 2012, ApJ, 747, 18

Viana Almeida, P., Santos, N. C., Melo, C., et al. 2009, A\&A, 501, 965

White, G. L., Jauncey, D. L., Batty, M. J., Peters, W. L., \& Gulkis, S. 1988, PASP, 100,825

Zuckerman, B., Song, I., \& Bessell, M. S. 2004, ApJ, 613, L65 